\title{
Effective properties of regular elastic laminated shell composite
}

\author{
D. Guinovart-Sanjuán a , R. Rodríguez-Ramos a, *, R. Guinovart-Díaz a , J. Bravo-Castillero a, \\ F.J. Sabina ${ }^{\text {b }}$, J. Merodio ${ }^{\mathrm{c}}$, F. Lebon ${ }^{\mathrm{d}}$, S. Dumont ${ }^{\mathrm{d}, \mathrm{e}}$, A. Conci ${ }^{\mathrm{f}}$ \\ ${ }^{a}$ Facultad de Matemática y Computación, Universidad de La Habana, San Lázaro y L, Vedado, La Habana CP 10400, Cuba \\ ${ }^{\mathrm{b}}$ Instituto de Investigaciones en Matemáticas Aplicadas y en Sistemas, Universidad Nacional Autónoma de México, Apartado Postal 20-126, \\ Delegación de Álvaro Obregón 01000, Mexico \\ ${ }^{\mathrm{c}}$ Departamento de Mecánica de los Medios Continuos y T. Estructuras, E.T.S. de Caminos, Canales y Puertos, Universidad Politécnica de Madrid, \\ C.P. 28040 Madrid, Spain \\ d Laboratoire de Mécanique et d'Acoustique, Aix-Marseille University, CNRS, UPR 7051, Centrale Marseille, 4 Impasse Nikola Tesla, CS 40006,13453 \\ Marseille Cedex 13, France \\ e Université de Nîmes, Institut de Mathématiques Alexander Grothendieck, CNRS UMR 5149, CC. 051, Pl. E. Bataillon, 34 095 Montpellier Cedex 5, France \\ ${ }^{\mathrm{f}}$ Instituto de Computação/Universidade Federal Fluminense (UFF), Av. Gal. Milton Tavares de Souza, s/n ${ }^{\circ}$, Niterói, CEP: $24210-346$ Rio de Janeiro, Brazil
}

\begin{abstract}
The manuscript offers a methodology to solve an analytical model of a heterogeneous elastic problem for curvilinear layered structures, using the two scales asymptotic homogenization method (AHM). The local problems and the mechanical properties of the local functions were derived. The analytical modeling for the linear elastic problem considering quasi-periodic multi-layered curvilinear composites and the corresponding homogenized problem were obtained. The analytic expression of the effective stress for curvilinear composites is presented. In order to validate the presented model, comparisons with a computational modeling and experimental results for Fibonacci laminated composite and wavy laminated structure are given. The methodology is applied to composites with thickness variation where the effective coefficients were computed and a comparison between the results reported by AHM and numerical analysis given by finite element method (FEM) is presented. Finally, the aorta is studied as a curvilinear laminated shell composite and the above results were used to determinate the effective elastic tensor for healthy and unhealthy aorta using AHM and FEM.
\end{abstract}

\section{Introduction}

The composite materials have been very popular for the last years due their exceptional mechanical properties. The laminated shell and sandwich composites had an important application in aerospace industry, automotive engineer [1-4] and textile manufacturing [5-7]. The composite conical shells have been widely used in various fields of technology as important structural components due to their special geometric shapes [8,9]. The use of the curvilinear composite structures in new engineering applications is significantly facilitated if the effective properties such as elastic, piezoelectic, thermo-elastic etc., can be predicted.

Homogenization is a useful mathematical method for solving boundary value problems in media with fine periodic structure. Two scale homogenization techniques have been used to solve

\footnotetext{
* Corresponding author. Tel.: +53 78790890.

E-mail addresses: reinaldo@matcom.uh.cu, rerora2006@gmail.com (R. Rodríguez-Ramos).
}

periodic heterogeneous problems [10-12]. Other homogenization method is related to the concept of H-convergence [13,14]. The fourth order heterogeneous constitutive tensor $\mathrm{H}$-converges to the effective tensor when the solution of the corresponding heterogeneous elasticity problem converges weakly to the effective displacement.

The development of new mathematical models and mathematic techniques helps to study the elastic properties of a laminated shell composite and bio-composite (cornea, aorta). Composites with periodic structure are often encountered in structural mechanics, in particular shell composites, [15-17]. The homogenization [10,18-21] and finite elements [7,22,23] techniques for shell are very used in these problems. Some recent works have been published related to effective properties of the smart composite shells $[12,18,19]$ and wavy laminated shell composite [13,24], among others. In particular, behaviors of bio-composites are being analyzed with mathematical shell methods. Three dimensional models of human cornea [25,26], and the aorta [27], are important motivation for this work. 
In this contribution, elastic composites in which the material coefficients are assumed to be rapidly oscillating periodic functions of a curvilinear coordinates system is studied and the two scales asymptotic homogenization method is used to solve the heterogeneous elastic problem. The novelty of this research is to obtain a homogenized problem with effective coefficients for a curvilinear laminated composites as extension of previous works [13,28-30]. Besides, the local problems and the general analytic expression for the effective coefficients are derived and the effective properties in Refs. $[10,13]$ are obtained as particular case. The results presented in Ref. [31] for a Fibonacci laminated composite are compared with the results obtained by AHM for a quasi periodic shell composite. The effective coefficients reported in Ref. [13] for two waviness layers composite were compared with a laminated wavy structure with soft/hard interface between the layers. As an example of stratified structure, a composite with thickness variation is considered and the effective coefficients were computed using AHM (Asymptotic Homogenization Methods) and FEM (Finite Elements Method). The paper gives a methodology to analyze the heterogeneous elastic problem in curvilinear structures. This methodology is used to determinate the effective properties of the aorta and analyze the artery as a laminated shell composite. Comparisons between the effective coefficients for healthy and unhealthy (due to the presence of plaques) artery is presented. The present model is validated with Finite Element Method.

\section{Elastic problem for curvilinear structure}

\subsection{Curvilinear structures}

In the present work, a quasi-periodic elastic structure is understood, if there is a coordinate system $\mathbf{x}=\left(x_{1}, x_{2}, x_{3}\right) \in \Omega \subset \mathbb{R}^{3}$ such that the operator $\boldsymbol{\sigma}=F(\boldsymbol{\varepsilon}, \mathbf{x}, \mathbf{y})$ who related stress $(\boldsymbol{\sigma})$ and strain $(\varepsilon)$ is regular in $\mathbf{x}$ and $\mathbf{Y}$-periodic in $\mathbf{y}$, where $\mathbf{y}=\mathbf{x} / \varepsilon \in \mathbf{Y}(\mathbf{Y}$ unit cell) and $\varepsilon$ is a very small parameter.

Additionally, for certain structure there is an oscillation in its geometrical configuration and a stratified function is used to describe the geometry of the composite [13]. Also, in this sense a variation of the thickness of the unit cell can be considered. A generalization of these ideas is presented in further sections.

In the case when the operator $\boldsymbol{\sigma}=F(\boldsymbol{\varepsilon}, \mathbf{x}, \mathbf{y})$ is regular in $\mathbf{x}$ and $\mathbf{Y}$ periodic in $\mathbf{y}$, where $\mathbf{y}=\varrho(\mathbf{x}) / \varepsilon$ and $\varrho: \mathbb{R}^{3} \rightarrow \mathbb{R}^{3}$, it defines an elastic curvilinear structure.

The particular case when the function $\mathbf{Q} \equiv \mathbf{I}$ (identity function) the curvilinear structure is a quasi-periodic structure. Another important example are the shell composites; for this one, the function $\varrho$, called stratified function, has the property $\varrho: \mathbb{R}^{n} \rightarrow \mathbb{R}^{p}$ where $n>p$, [13], see Fig. 1 .

\subsection{Statement of the elastic problem for curvilinear structures}

Consider a certain curvilinear heterogeneous structure $\Omega$ bounded by $\Sigma=\Sigma_{1} \cup \Sigma_{2}$ in the coordinate system $\mathbf{x}=\left(x_{1}, x_{2}, x_{3}\right)$ and a periodic function $\mathbf{Q}=\left(\varrho_{1}(\mathbf{x}), \varrho_{2}(\mathbf{x}), \varrho_{3}(\mathbf{x})\right)$. The aforementioned operator $F$ in this structure is the Hooke's law

$\boldsymbol{\sigma}_{\varepsilon}=\mathbf{C}_{\varepsilon}\left(\frac{\mathbf{Q}(\mathbf{x})}{\varepsilon}, \mathbf{x}\right): \boldsymbol{\varepsilon}_{\varepsilon}$

where $\mathbf{C}_{\varepsilon}$ is regular in $\mathbf{x}$ and $\mathbf{Y}$-periodic in $\mathbf{y}$, where $\mathbf{y}=\mathbf{\varrho}(\mathbf{x}) / \varepsilon$ is the fast curvilinear coordinate system.
The equilibrium equation of the elastic theory has the following expression

$\operatorname{div} \boldsymbol{\sigma}_{\varepsilon}+\mathbf{f}=0 \quad$ on $\quad \Omega$

with boundary conditions

$\mathbf{u}^{\varepsilon}=\mathbf{u}^{0}$ on $\Sigma_{1}, \quad \boldsymbol{\sigma}^{\varepsilon} \cdot \mathbf{n}=\mathbf{S}$ on $\Sigma_{2}$,

where $\mathbf{u}^{\varepsilon}$ is the displacement, $\mathbf{u}^{0}$ is the known displacement on $\Sigma_{1}, \mathbf{S}$ is the stress vector on $\Sigma_{2}$ and $\mathbf{n}$ is the external normal vector of $\Sigma_{2}$.

The coordinate system $\mathbf{x}$ is curvilinear and considering the Einstein's summation rule, where the Latin index run from 1 to 3 , the expression of the equilibrium equation can be written

$\sigma^{i j} \|_{j}+f_{i}=\sigma_{j}^{i j}+\Gamma_{j k}^{i} \sigma^{k j}+\Gamma_{j k}^{j} \sigma^{i k}+f^{i}=0$ on $\Omega$,

where $\{\cdot\} \|_{j}$ denotes the covariant derivate, $\{\cdot\}_{j}=\frac{\partial}{\partial x_{j}}\{\cdot\}$ the derivation respect to the slow or global curvilinear coordinate and $\Gamma_{j k}^{i}$ the Christoffel's symbols of second type.

Considering the Cauchy's formula, who related the strains and the displacements $\varepsilon_{m n}=1 / 2\left(u_{m}\left\|_{n}+u_{n}\right\|_{m}\right)$ and substituting into (1) yields

$\sigma^{i j}=C^{i j m n} u_{m} \|_{n}=C^{i j m n}\left(u_{m, n}-\Gamma_{m n}^{k} u_{k}\right)$.

Replacing (5) into (4), the generalized equilibrium problem of elasticity theory takes the following expression

$$
\begin{aligned}
& \left(\frac{\varrho_{k, j}}{\varepsilon} C_{\mid k}^{i j m n}+C_{, j}^{i j m n}+\Gamma_{j k}^{\mathrm{i}} C^{k j m n}+\Gamma_{j k}^{j} C^{i k m n}\right)\left(u_{m, n}-\Gamma_{m n}^{r} u_{r}\right) \\
& +C^{i j m n}\left(u_{m, n j}-\Gamma_{m n, j}^{r} u_{r}-\Gamma_{m n}^{r} u_{r, j}\right)+f^{i}=0 \text { on } \Omega,
\end{aligned}
$$

with boundary conditions

$u_{i}=u_{i}^{0}$ on $\Sigma_{1} \quad\left(C^{i j m n}\left(u_{m, n}-\Gamma_{m n}^{r} u_{r}\right)\right) n_{j}=S^{i}$ on $\Sigma_{2}$,

where $\{\cdot\}_{\mid j}=\frac{\partial}{\partial y_{j}}\{\cdot\}$ denotes the derivation respect to the fast or local curvilinear coordinate.

\section{Asymptotic homogenization method}

In order to solve the problem (6) and (7) with fast oscillating coefficients, the two scales Asymptotic Homogenization Method (AHM) is used.

The general asymptotic expansion is

$u_{m}^{(\varepsilon)}=v_{m}+\varepsilon \cdot v_{l}\left\|_{k} N_{m}^{l k}+\varepsilon^{2} \cdot \neq v_{l}\right\|_{k} j N_{m}^{l k j}+\ldots$,

where $v_{m} \equiv v_{m}(\mathbf{x})$, and the local functions $N_{m}^{l k \ldots} \equiv N_{m}^{l k \ldots}(\mathbf{x}, \mathbf{y})$ are $\mathbf{y}$ periodic functions and $\left\langle N_{m}^{l k \ldots}\right\rangle=0$, where $\langle\varphi\rangle=\frac{1}{\mathbf{V}_{\mathbf{Y}}} \int_{\mathbf{Y}} \varphi \sqrt{g} d y ; \mathbf{V}_{\mathbf{Y}}$ volume of $\mathbf{Y}, \sqrt{g}=\sqrt{\operatorname{det}\left[g_{i j}\right]}$ and $\left[g_{i j}\right]$ is the metric tensor [16].

In order to obtain the effective coefficients, the following expansion is proposed, 

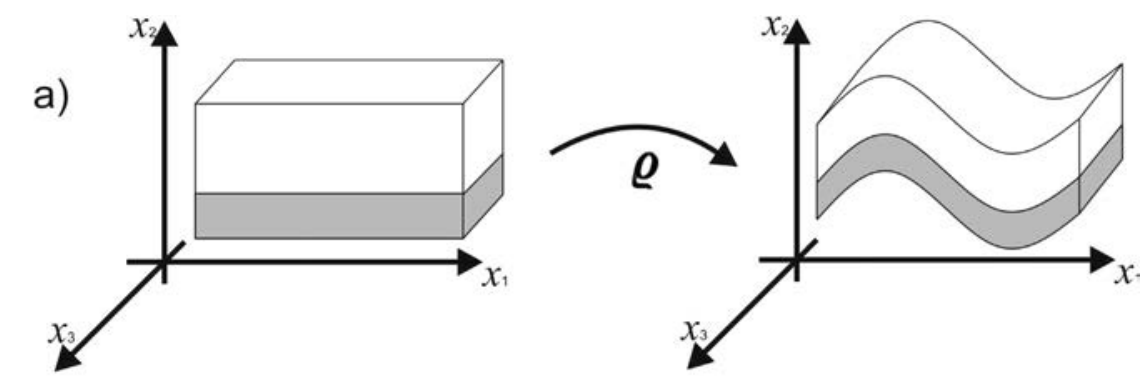

b)
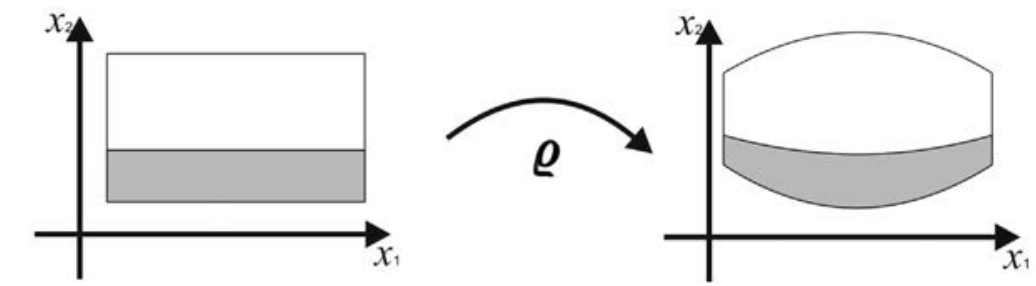

Fig. 1. Laminated shell structures and the transformation $\mathrm{o}$ : a) unit cell with a wavy transformation, b) unit cell with a thickness variation

$u_{m}^{(\varepsilon)}=v_{m}+\varepsilon \cdot v_{l} \|_{k} N_{m}^{l k}+o(\varepsilon)=v_{m}+\varepsilon\left(v_{l, k}-\Gamma_{l k}^{p} v_{p}\right) N_{m}^{l k}+o(\varepsilon)$,

$u_{m}^{(\varepsilon)}=v_{m}+\varepsilon\left[N_{(0) m}^{p} v_{p}+N_{(1) m}^{l k} v_{l, k}\right]+o(\varepsilon)$,

where $N_{(1) m}^{l k} \equiv N_{m}^{l k}$ is the local function for the first order approach and $N_{(0) m}^{p}=-\Gamma_{l k}^{p} N_{(1) m}^{l k}$.

Replacing (9) into the equilibrium Equation (6), grouping and analyzing the coefficients for different powers of the parameter $\varepsilon$, a sequence of problems is obtained. From the continuity of (9), the coefficient of $\varepsilon^{-1}$ is equating to zero and yields the following two local problems on $\mathbf{Y}$

$\left(\varrho_{q, j} C^{i j l k}+\varrho_{p, n} C^{i j m n} N_{(1) m \mid p}^{l k} \varrho_{q, j}\right)_{\mid q}=0$

$\left(-\varrho_{l, j} C^{i j m n} \Gamma_{m n}^{p}+\varrho_{l, j} C^{i j m n} \varrho_{t, n} N_{(0) m \mid t}^{p}\right)_{\mid l}=0$.

These two local problems are related considering the fact that $N_{(0) m}^{p}=-\Gamma_{l k}^{p} N_{(1) m}^{l k}$.

Applying the average operator to the coefficient of $\varepsilon^{0}$ and grouping the coefficients of $v_{k, j l}, v_{k, l}$ and $v_{l}$ the general expression of the effective coefficients are obtained

$C_{e}^{i j k l} \equiv \widehat{h}^{i j k l}=\left\langle C^{i j k l}+C^{i j m n} \varrho_{p, n} N_{(1) m \mid p}^{k l}\right\rangle$.

In general the effective coefficients (12) are functions that depend on $\mathbf{x}$ through $\varrho_{p, n}$. The Equation (12) coincides with the effective coefficient reported in Ref. [21] for $\varrho_{p, n}=\delta_{p n}$.

The homogenized coefficients for the elastic problem are

$\widehat{h}^{i l k}=\widehat{h}_{j}^{i j l k}-\Gamma_{m n}^{l} \widehat{h}^{i k m n}+\Gamma_{j n}^{i} \widehat{h}^{n j l k}+\Gamma_{j n}^{j} \widehat{h}^{i n l k}$, $\widehat{h}^{i l}=-\left(\Gamma_{m n}^{l} \widehat{h}^{i j m n}\right)_{j}-\left(\Gamma_{j k}^{i} \widehat{h}^{k j m n}+\Gamma_{j k}^{j} \widehat{h}^{i k m n}\right) \Gamma_{m n}^{l}$,

$\widehat{h}_{*}^{i j l}=-\Gamma_{m n}^{l} \widehat{h}^{i j m n}$.

A particular form for the homogenized coefficients is obtained from (13)-(15) when the periodicity function $\mathrm{\varrho}\left(x_{1}, x_{2}, x_{3}\right)$ is the identity. In this case

$\widehat{h}^{i k l}=-\widehat{h}^{i k m n} \Gamma_{m n}^{l}+\left(\Gamma_{j r}^{i} \widehat{h}^{r j l k}+\Gamma_{j r}^{j} \widehat{h}^{i r l k}\right)$,

$\widehat{h}^{i l}=-\widehat{h}^{i j m n} \Gamma_{m n, j}^{l}-\left(\Gamma_{j r}^{i} \widehat{h}^{j r m n}+\Gamma_{j r}^{j} \widehat{h}^{r i m n}\right) \Gamma_{m n}^{l}$,

$\widehat{h}_{*}^{i j l}=-\widehat{h}^{i j m n} \Gamma_{m n}^{l}$.

The results given by (16)-(18), are obtained in Ref. [10] for quasi-periodic structures.

The homogenized coefficients (16)-(18) are zero in case of a rectangular coordinate system, because $\Gamma_{i j}^{l}=0$, for all $l, i, j$.

An equivalent problem to (6) and (7) with constant coefficients can be formulated using the results obtained in (12)-(15),

$\widehat{h}^{i l} v_{l}+\widehat{h}^{i k l} v_{[l, k]}+\widehat{h}^{i m l n} v_{l, n m}+f_{i}=0$ on $\Omega$,

with boundary conditions

$v_{i}=u_{i}^{0}$ on $\Sigma_{1} \quad\left(\widehat{h}_{*}^{i j l} v_{l}+\widehat{h}^{i j l k} v_{l, k}\right) n_{j}=S^{i}$ on $\Sigma_{2}$,

where $v_{[l, k]}=1 / 2\left(v_{k, l}+v_{l, k}\right)$. 
The problem (19) and (20) is called the homogenized problem and the notation " $\widehat{h}$ " means the homogenized coefficients for the curvilinear structures.

Substituting the Equations (12), (16) and (17) into (19) the following equation is obtained

$\sigma_{e}^{i j} \|_{j}+f_{i}=0$

where

$\sigma_{e}^{i j}=\left\langle C^{i j m n} \varrho_{p, n} N_{(1) m \mid p}^{k l}+C^{i j k l}\right\rangle v_{k, l}+\left\langle C^{i j m n} \varrho_{p, n} N_{(0) m \mid p}^{r}-\Gamma_{m n}^{r} C^{i j m n}\right\rangle v_{r}$.

The expression (22) is rewritten taking into account the relation $N_{(0) m}^{p}=-\Gamma_{l k}^{p} N_{(1) m}^{l k}$ and Equation (12)

$$
\begin{aligned}
\sigma_{e}^{i j} & =\left\langle C^{i j m n} \varrho_{p, n} N_{(1) m \mid p}^{k l}+C^{i j k l}\right\rangle v_{k, l}-\Gamma_{k l}^{r}\left\langle C^{i j m n} \varrho_{p, n} N_{(1) m \mid n}^{k l}+C^{i j k l}\right\rangle v_{r} \\
& =\left\langle C^{i j m n} \varrho_{p, n} N_{(1) m \mid p}^{k l}+C^{i j k l}\right\rangle\left(v_{k, l}-\Gamma_{k l}^{r} v_{r}\right) \\
& =C_{e}^{i j k l} v_{k} \|_{l} .
\end{aligned}
$$

\section{Curvilinear structures with isotropic layers}

Now a laminated curvilinear structure is considered, where the geometry is described using a curvilinear coordinate system with orthogonal components. In this case the metric tensor has the expression

$$
\left[g_{i j}\right]=\left[\begin{array}{ccc}
g_{11} & 0 & 0 \\
0 & g_{22} & 0 \\
0 & 0 & g_{33}
\end{array}\right] .
$$

Now we assume that the elastic components of the laminated curvilinear structure are isotropic. Thus the elastic tensor can be written as

$C^{i j k l}=\lambda g^{i j} g^{k l}+\mu\left(g^{i k} g^{j l}+g^{i l} g^{j k}\right)$,

where $\left[g^{i j}\right]=\left[g_{i j}\right]^{-1}$. In case of rectangular coordinate system, the expression (25) is the usual elastic tensor [16].

Besides, for the stratified laminated composites the periodicity (stratified) function $\varrho$ has the property: $\varrho: \mathbb{R}^{m} \rightarrow \mathbb{R}^{1}$ with $m=2,3$ (see Fig. 1) [13].

\subsection{Curvilinear coordinates and the projection on $x_{3}$}

As an illustration of the above mentioned, we consider a certain orthogonal curvilinear coordinate system. The elastic tensor is obtained from the Equations (24) and (25). Now, the stratified function as the projection $\varrho:\left(x_{1}, x_{2}, x_{3}\right) \rightarrow x_{3}$ and substituting into Equation (12), the following nonzero coefficients are obtained

$$
\begin{aligned}
C_{e}^{1111}= & \left\langle C^{1111}\right\rangle+\left\langle\frac{C^{1133}}{C^{3333}}\right\rangle^{2}\left\langle\left(C^{3333}\right)^{-1}\right\rangle^{-1}-\left\langle\frac{\left(C^{1133}\right)^{2}}{C^{3333}}\right\rangle, \\
C_{e}^{1122}= & \left\langle C^{1122}\right\rangle+\left\langle\frac{C^{1133}}{C^{3333}}\right\rangle\left\langle\left(C^{3333}\right)^{-1}\right\rangle^{-1}\left\langle\frac{C^{2233}}{C^{3333}}\right\rangle \\
& -\left\langle\frac{C^{1133} C^{2233}}{C^{3333}}\right\rangle, \\
C_{e}^{1133}= & \left\langle C^{1133}\left(C^{3333}\right)^{-1}\right\rangle\left\langle\left(C^{3333}\right)^{-1}\right\rangle^{-1}, \\
C_{e}^{2233}= & \left\langle C^{2233}\left(C^{3333}\right)^{-1}\right\rangle\left\langle\left(C^{3333}\right)^{-1}\right\rangle^{-1}, \\
C_{e}^{2222}= & \left\langle C^{2222}\right\rangle^{+}\left\langle C^{2233}\left(C^{3333}\right)^{-1}\right\rangle^{2}\left\langle\left(C^{3333}\right)^{-1}\right\rangle \\
& -\left\langle\left(C^{2233}\right)^{2}\left(C^{3333}\right)^{-1}\right\rangle, \\
C_{e}^{3333}= & \left\langle\left(C^{3333}\right)^{-1}\right\rangle^{-1} C_{e}^{2323}=\left\langle\left(C^{2323}\right)^{-1}\right\rangle^{-1} \\
C_{e}^{1313}= & \left\langle\left(C^{1313}\right)^{-1}\right\rangle^{-1}, C_{e}^{1212}=\left\langle C^{1212}\right\rangle^{-1} .
\end{aligned}
$$

This result is the same to the Formula (1.22) page 149 presented in Ref. [10], for orthotropic composites (9 elastic constant). The effective coefficients for a composite with isotropic components are a particular case of the Equation (26), see formula (1.19) page 147 of [10].

\subsection{Curvilinear orthogonal coordinates and a general form of the stratified function}

Now we consider the case when the elastic tensor $\boldsymbol{C} \equiv \boldsymbol{C}\left(\frac{\varrho}{\varepsilon}\right)$ is given by the expression (25) for an orthogonal coordinate system, see Equation (24), and the stratified function is $\varrho: \mathbb{R}^{3} \rightarrow \mathbb{R}$, $\varrho \equiv \varrho\left(x_{1}, x_{2}, x_{3}\right)$. Substituting this expression of $\varrho$ into (12) and using the Voigt notation, the following equation is obtained

$$
\begin{aligned}
C_{e}^{i j}=\langle & C^{i j}+\left(C^{i 1} \frac{\partial \varrho}{\partial x_{1}}+C^{i 6} \frac{\partial \varrho}{\partial x_{2}}+C^{i 5} \frac{\partial \varrho}{\partial x_{3}}\right) \frac{\partial N_{1}^{j}}{\partial y} \\
& +\left(C^{i 6} \frac{\partial \varrho}{\partial x_{1}}+C^{i 2} \frac{\partial \varrho}{\partial x_{2}}+C^{i 4} \frac{\partial \varrho}{\partial x_{3}}\right) \frac{\partial N_{2}^{j}}{\partial y} \\
& \left.+\left(C^{i 5} \frac{\partial \varrho}{\partial x_{1}}+C^{i 4} \frac{\partial \varrho}{\partial x_{2}}+C^{i 3} \frac{\partial \varrho}{\partial x_{3}}\right) \frac{\partial N_{3}^{j}}{\partial y}\right\rangle .
\end{aligned}
$$

The Equation (27) is a generalization of the results presented in Refs. [10,13] (for instance, see formula (3.35) in Ref. [13]).

\section{Application of AHM to a quasi-periodic structure with isotropic layers}

\subsection{Layer composite with Fibonacci structure}

In order to validate the present model, a Fibonacci laminated composite is studied. The Fibonacci structure can be considered as a quasi-periodic structure, [31]. There are two basic elements $L_{1}$ and $L_{2}$, where each of them are subdivided into two layers. Each layer $L_{i}$ $(i=1,2)$ is made of Aluminum ( $\mathrm{Al})$ and Tantalum (Ta) with thickness 
$\mathrm{d}_{i}^{\mathrm{Al}}$ and $\mathrm{d}_{i}^{\mathrm{Ta}}$ respectively. Moreover, the thicknesses of $L_{1}$ and $L_{2}$ are $\mathrm{d}_{1}=\mathrm{d}_{1}^{\mathrm{Al}}+\mathrm{d}_{1}^{\mathrm{Ta}}=46.6 \AA$ and $\mathrm{d}_{2}=\mathrm{d}_{2}^{\mathrm{Al}}+\mathrm{d}_{2}^{\mathrm{Ta}}=29.6 \AA$ respectively. The associated quasi-periodicity is $\mathrm{d}=\tau d_{1}+d_{2}=105 \AA$. In Table 1 a comparison with theoretical and experimental results reported in Ref. [31] for quasi-periodic Fibonacci superlattices (QPFSL) is given. A good coincidence between the numerical (QPFSL) and experimental (Exp.) results with the results obtained by the present model (AHM) can be observed.

\subsection{Effective coefficients for a wavy laminated composite}

As a particular case we considered a wavy bimetallic laminated composite. The Cartesian coordinate system is used to describe the geometry of this composite. The mechanical properties are discontinuous in the composite.

Also, we consider an interface between the layers. This interface can be considered as a third layer of the composite. The effective coefficients can be calculated for a shell structure with three different layers of thickness denoted by $t_{1}, t_{2}$ and $t_{3}$ respectively (see Fig. 2).

The materials used in the composite are stainless steel (with Young's modulus, $E_{1}=206.74 \mathrm{GPa}$, Poisson ratio, $\nu_{1}=0.3$ ) and aluminum (with Young's modulus $E_{3}=72.04 \mathrm{GPa}$, Poisson ratio $\nu_{3}=0.35$ ). In order to compare the behavior of the effective coefficients with the interface and the effective coefficients presented in Ref. [13] for a wavy structure without interface, four cases are considered. The volume fraction of the layers $t_{1}$ (Stainless steel), $t_{2}$ (interface), $t_{3}$ (Aluminum), the values of Young's modulus and Poisson's ratio in the interface $t_{2}$ are given in Table 2 .

The volume fraction $V_{t_{2}}=0$ means no interface between the layers for the structure presented in Ref. [13], and therefore the volume $V_{t_{1}}=0.2$ and $V_{t_{3}}=0.8$.

The cases 1 and 2 are related to hard interface with different volume fraction between the layers (interface volume fraction 1\% and $5 \%$ of the unit cell respectively) and the elastic properties of the interface are stronger than the other two layers. The cases 3 and 4 correspond to a soft interface with different volume fraction and weaker elastic properties for the interface.

The wavy behavior is described by the stratified function

$\varrho\left(x_{1}, x_{2}\right)=x_{2}-H \sin \left(\frac{2 \pi x_{1}}{L}\right)$,

where $H$ is a parameter related to the oscillation, $L$ is the length of the unit cell [13] (see Fig. 2), and the variation of $x_{3}$ is not considered.

Considering Cartesian coordinate system for solving the local problem (10), the local functions are obtained and replacing them into (27), the effective coefficients (with interface, cases $1-4$, and without interface as it is considered in Ref. [13]) are computed.

The results presented in Fig. 3 show the behavior of the aforementioned laminated shell composite with waviness, when hard and soft interfaces between the layers are considered. Also a comparison with one of the particular cases presented in Ref. [13] $(H / L=0.25)$ is shown. The hard interface reinforces the effective

Table 1

Comparison of the effective coefficients obtained by the present model (AHM) with experimental and numerical results of [31].

\begin{tabular}{llllll}
\hline Methods & $C_{e}^{11}$ & $C_{e}^{33}$ & $C_{e}^{44}$ & $C_{e}^{12}$ & $C_{e}^{13}$ \\
\hline QPFSL & 162.0 & 142.0 & 31.0 & 80.6 & 71.2 \\
AHM & 162.0545 & 142.0856 & 31.0558 & 80.7079 & 71.1881 \\
Expt. & $167 \pm 7$ & & $38 \pm 0.5$ & & \\
\hline
\end{tabular}

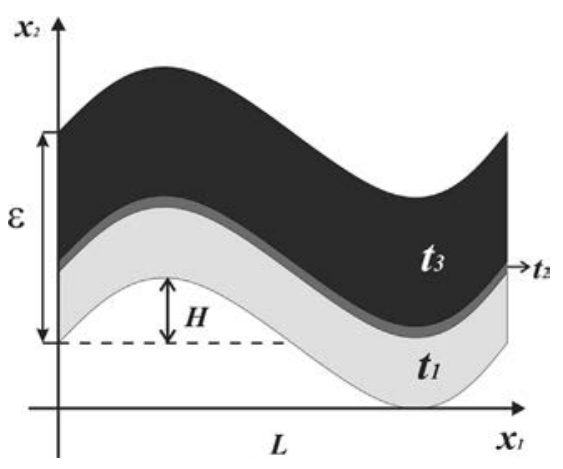

Fig. 2. Structure with waveny behavior.

properties as can be observed in the coefficients $C_{e}^{11}, C_{e}^{55}$ and $C_{e}^{66}$. However, contrary effect occurs for $C_{e}^{12}$.

\subsection{Laminated composite with variation on the thickness}

Now we consider a laminated composite where the thickness of the layers is different at some points of the unit cell. The structure is composed by two materials: stainless steel (Young's modulus, $E_{1}=206.74 \mathrm{GPa}$, Poisson ratio, $\nu_{1}=0.3$ ) and aluminum (Young's modulus $E_{3}=72.04 \mathrm{GPa}$, Poisson ratio $\nu_{3}=0.35$ ) with volume fraction $V_{t_{1}}=0.2$ and $V_{t_{2}}=0.8$.

The surface $\varrho\left(x_{1}, x_{2}\right) / \varepsilon=\left(x_{2}-0.5 x_{2} x_{1}^{2}\right) / \varepsilon$ for $x_{1}, x_{2} \in[-1,1]$ defines the unit cell. The projections of the curves

$\varrho\left(x_{1}, x_{2}\right)=x_{2}-0.5 x_{2} x_{1}^{2}=$ const,

into the plane $x_{1} y$ show the thickness variation of the composite, [13]. In $x_{2}$ direction the stiffness properties have fast oscillating behavior. Then for every value of $x_{2} \in[-1,1]$ we have a value function of the properties, with $x_{1}$ dependence, see Fig. $1 \mathrm{~b}$.

The variable $y$ varies between the curves $y_{1}=\left(-1+0.5 x_{1}^{2}\right) / \varepsilon$ and $y_{2}=\left(1-0.5 x_{1}^{2}\right) / \varepsilon$. The curve $y_{3}=\left(-0.6+0.3 x_{1}^{2}\right) / \varepsilon$ separates the two material layers. The function (28) describes the thickness variation of the unit cell. Variation of the elastic properties in $x_{3}$ direction is not considered, thus the elastic properties have rapidly oscillation in $y$ direction.

The effective coefficients can be calculated by solving the local problem (10) and using the equations presented in Appendix A. In this particular case the average operator $\langle\cdot\rangle=V_{t_{1}}(\cdot)_{1}+V_{t_{2}}(\cdot)_{2}$.

In order to validate the model, we compare the values of the coefficients $C_{e}^{11}, C_{e}^{16}, C_{e}^{55}$ and $C_{e}^{66}$ for the thickness variation function (28) with the values $x_{2}=-1$ and $x_{1}=[-1,-0.5,0,0.5,1]$ (see Table 3); $x_{1}=-1$ and $x_{2}=[-1,-0.5,0,0.5,1]$ (see Table 4 ). The methods used for the comparison are the Asymptotic Homogenization Method (AHM) and Finite Element Method (FEM). The results computed with FEM are obtained solving Equation (10) with the classical Finite Element Method [32-34] that provides the local functions $N_{(1) m}^{l k}$. Then, the effective coefficients are calculated using (12).

Table 2

Volume fraction of the layers $t_{1}, t_{2}, t_{3}$, the values of Young's modulus $E_{2}$ and Poisson's ratio $\nu_{2}$ in the interface $t_{2}$

\begin{tabular}{llllll}
\hline Case & $V_{t_{1}}$ & $V_{t_{2}}$ & $V_{t_{3}}$ & $E_{2}$ & $\nu_{2}$ \\
\hline 1 & 0.198 & 0.01 & 0.792 & 310.11 & 0.35 \\
2 & 0.19 & 0.05 & 0.76 & 310.11 & 0.35 \\
3 & 0.198 & 0.01 & 0.792 & 20.67 & 0.35 \\
4 & 0.19 & 0.05 & 0.76 & 20.67 & 0.35 \\
\hline
\end{tabular}



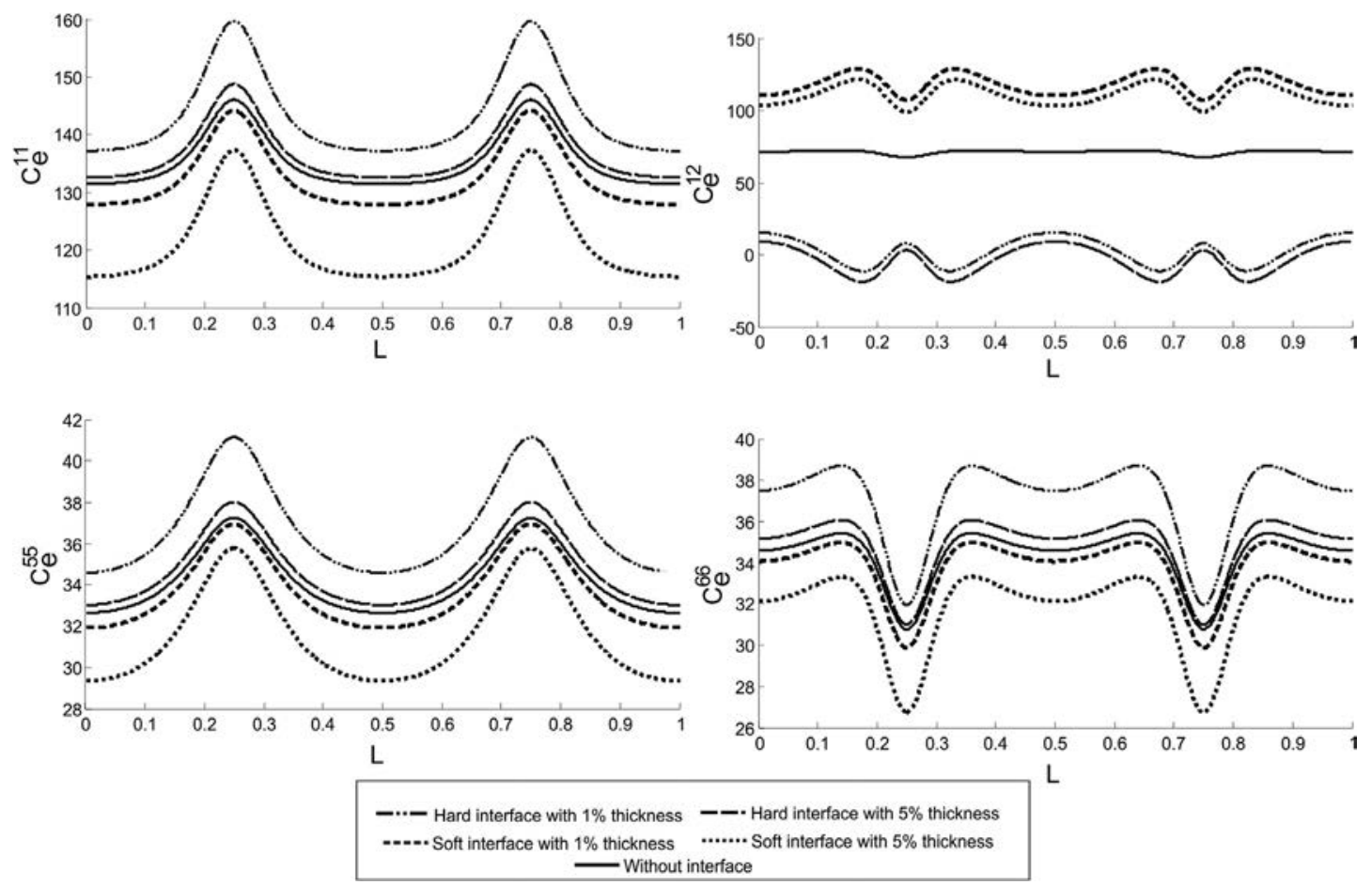

Fig. 3. Comparison between the effective coefficients $C_{e}^{11}, C_{e}^{12}, C_{e}^{55}$ and $C_{e}^{66}$ with oscillating ratio $H / L=0.25$ for different properties of the interface and the composite without interface reported by Ref. [13].

Table 3

Comparison between AHM and FEM of the effective coefficients $C_{e}^{11}, C_{e}^{16}, C_{e}^{55}$ and $C_{e}^{66}$ for different values of $x_{1}$ and for $x_{2}=-1$.

\begin{tabular}{|c|c|c|c|c|c|c|c|c|}
\hline \multirow[t]{2}{*}{$x_{1}$} & \multicolumn{2}{|l|}{$C_{e}^{11}$} & \multicolumn{2}{|l|}{$C_{e}^{16}$} & \multicolumn{2}{|l|}{$C_{e}^{55}$} & \multicolumn{2}{|l|}{$C_{e}^{66}$} \\
\hline & AHM & FEM & AHM & FEM & AHM & FEM & AHM & FEM \\
\hline-1 & 130.967 & 130.967 & 0.786 & 0.786 & 32.066 & 32.066 & 33.753 & 33.753 \\
\hline-0.5 & 138.861 & 138.861 & 5.294 & 5.294 & 35.654 & 35.654 & 34.230 & 34.230 \\
\hline 0 & 146.040 & 146.040 & 0 & 0 & 37.248 & 37.248 & 30.771 & 30.771 \\
\hline 0.5 & 138.861 & 138.861 & -5.294 & -5.294 & 35.654 & 35.654 & 34.230 & 34.230 \\
\hline 1 & 130.967 & 130.967 & -0.786 & -0.786 & 32.066 & 32.066 & 33.753 & 33.753 \\
\hline
\end{tabular}

Table 4

Comparison by AHM and FEM of the effective coefficients $C_{e}^{11}, C_{e}^{16}, C_{e}^{55}$ and $C_{e}^{66}$ for different values of $x_{2}$ and for $x_{1}=-1$.

\begin{tabular}{|c|c|c|c|c|c|c|c|c|}
\hline \multirow[t]{2}{*}{$x_{2}$} & \multicolumn{2}{|l|}{$C_{e}^{11}$} & \multicolumn{2}{|l|}{$C_{e}^{16}$} & \multicolumn{2}{|l|}{$C_{e}^{55}$} & \multicolumn{2}{|l|}{$C_{e}^{66}$} \\
\hline & AHM & FEM & AHM & FEM & AHM & FEM & AHM & FEM \\
\hline-1 & 130.967 & 130.967 & 0.786 & 0.786 & 32.066 & 32.066 & 33.753 & 33.753 \\
\hline-0.5 & 133.823 & 133.823 & 3.778 & 3.778 & 34.009 & 34.009 & 35.431 & 35.431 \\
\hline 0 & 146.040 & 146.040 & 0 & 0 & 37.248 & 37.248 & 30.771 & 30.771 \\
\hline 0.5 & 133.823 & 133.823 & -3.778 & -3.778 & 34.009 & 34.009 & 35.431 & 35.431 \\
\hline 1 & 130.967 & 130.967 & -0.786 & -0.786 & 32.066 & 32.066 & 33.753 & 33.753 \\
\hline
\end{tabular}

We can appreciate good approximation between the results calculated by AHM and FEM. The difference of the values reported in Tables 3 and 4 is lower than $10^{-3}$.

\subsection{Application of the AHM to the aorta as a quasi-periodic composite}

The aorta is a layered bio-composite. In Ref. [27], the initial stress state, crack initiation and propagation in the intimal layer of aorta under multiple static loading conditions is investigated. In that process, three dimensional linear elastic isotropic models of human aorta were numerically analyzed to assess the stress state due to multiple loads, influence of stenosis rate on crack initiation, and finally crack growth pattern in the intimal layer due to change in material fracture resistance. In order to predict the stress state, tear initiation and propagation in the human aorta, computational modeling using finite element method was performed. The crack initiation in the intima layer due to the presence of plaque, under physiological loading conditions can be simulated. In this case a three layered model is developed with thickness of intima, media and adventitia layers varying at a ratio of 1:6:3 [35]. A cross section of the aorta has a $R_{1}$ inner radius of $1.02 \mathrm{~cm}$ and $R_{2}$ outer radius of $1.22 \mathrm{~cm}$. In this case a three layered model (healthy aorta) was developed with thickness of intima $\left(t_{1}=0.02 \mathrm{~cm}\right)$, media $\left(t_{2}=0.12 \mathrm{~cm}\right)$ and adventitia $\left(t_{3}=0.06\right)$. In addition to this, $40 \%$ stenosis (percentage of volume occupied by the plaque) was included. This situation can be interpreted as an aorta with obstruction (unhealthy aorta) due to the presence of plaques surrounded intima layer with thickness $t_{0}=0.4$ (four layers). The inner radius for the obstruction layer is reduced to $R_{0}=0.62$ (see Fig. 4). Stress variations in the aorta being the major cause of tear initiation

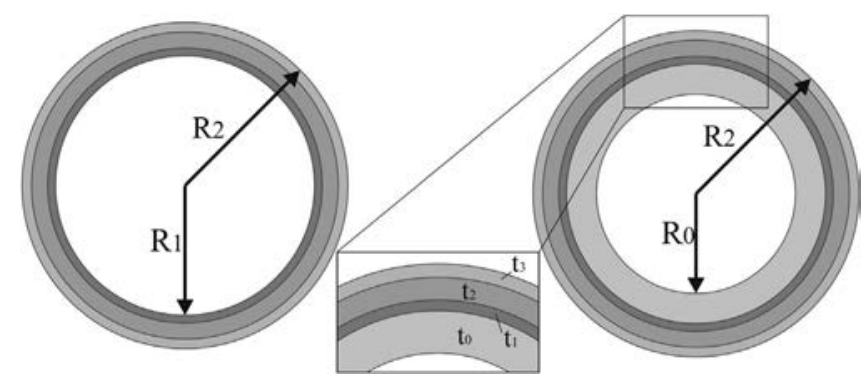

Fig. 4. Aorta model and the thickness $t_{0}$-plaque, $t_{1}$-intima, $t_{2}$-media and $t_{3}$-adventitia for each layer. 
in case of aneurysm. The region between the junction of brachiocephalic artery and the aorta trunk, aortic root is the most vulnerable location for the crack initiation.

The analysis of the stress state, crack initiation and some others phenomenon in heterogeneous media like aorta is complicated. In order to facilitate the ulterior study of such problems, we apply the asymptotic homogenization method to the aorta as a cylindrical laminated shell composite and an equivalent homogeneous elastic problem with effective properties is obtained.

The material properties in all the cases are considered as linear elastic isotropic, and the constants utilized are shown in Table 5.

The mechanical properties of each layer are presented in Table 5.

We consider the cylindrical coordinate system to describe the aorta, $\mathbf{x}=(\theta, z, r)$. The related metric tensor (24) has the following expression

$\left[g_{i j}\right]=\left[\begin{array}{lll}r^{2} & 0 & 0 \\ 0 & 1 & 0 \\ 0 & 0 & 1\end{array}\right]$.

For this case, the non vanishing components of the tensor $\left[g^{i j}\right]$ are $g^{11}=1 / r^{2}, g^{22}=1, g^{33}=1$.

The strain and the stress are functions of the variable $r$. The average operator is

$\langle F\rangle=\frac{1}{V_{Y}} \int_{Y} F \cdot \sqrt{g} d Y$.

where $g=\operatorname{det}\left[g_{i j}\right]=r^{2}($ see $(29))$. For the aorta, the average operator (30) is

$\langle F\rangle=\frac{2}{R_{2}^{2}-R_{\alpha}^{2}} \int_{R_{\alpha}}^{R_{2}} F \cdot r d r$,

where $\alpha=1$ for three layers and $\alpha=0$ for the case of the obstruction (four layers).

The nonzero Christoffel's symbols are

$\Gamma_{11}^{3}=-r, \quad \Gamma_{13}^{1}=\Gamma_{31}^{1}=\frac{1}{r}$.

The general expression for the elastic tensor can be obtained from the Equation (25). The variation of the material properties occurs on $x_{3}=r$ direction. Using (26) the effective properties in the short index notation are shown in Table 6. Moreover, a comparison

Table 5

Mechanical properties of the aorta.

\begin{tabular}{lll}
\hline Layers & Young's modulus & Poisson's ratio \\
\hline Plaque & 0.2 & 0.49 \\
Intima & 2.98 & 0.49 \\
Media & 8.95 & 0.49 \\
Adventitia & 2.98 & 0.49 \\
\hline
\end{tabular}

with FEM is reported in this table for three layers (healthy aorta) with $\mathrm{N}=1000$ and four layers (unhealthy aorta) $\mathrm{N}=5000$ points, where $\mathrm{N}$ is the number of nodes for the discretization of the integration region. It is necessary to take $\mathrm{N}$ large enough because the periodicity condition only at a discrete level is imposed.

Using AHM the Equations (16) and (17) and the effective coefficients (Table 6) for three layered model and the coefficients of the homogenized problem (19) can be obtained. In general, the nonzero third order homogenized coefficients $\widehat{h}^{113}, \widehat{h}^{131}, \widehat{h}^{223}$, $\widehat{h}^{232}, \widehat{h}^{13}, \widehat{h}^{311}, \widehat{h}^{322} \widehat{h}^{333}$ and the nonzero second order homogenized coefficients $\widehat{h}^{11}, \widehat{h}^{33}$ can be compute. In Table 7 for example, only the third order coefficients $\widehat{h}^{131}, \widehat{h}^{223}$ and the second order coefficients $\widehat{h}^{11}$ and $\widehat{h}^{33}$ are shown for the positions $r=[1.02,1.06$, $1.1,1.14,1.18,1.22]$.

Using the effective coefficients (12) computed in Table 6 and the homogenized coefficients derived in Equations (16)-(18), the homogenized problem (19) under certain particular boundary conditions (20) can be solved and the displacement function $\mathbf{v}=\left(v_{1}, v_{2}, v_{3}\right)$ is calculated. Therefore, the effective stress (23) for the aorta as an homogeneous body can be determinate and consequently the behavior of the aneurysm and crack can be predicted as solution of an appropriate boundary value problem.

\section{Conclusions}

In this paper the analytic homogenization of elastic composites with generalized periodicity is presented. The two scale asymptotic homogenization method is used to determinate the local problem and the analytic expression for the effective coefficients. The general expression of the stress for a curvilinear structure is derived and the homogenized problem is stated. Three engineering microstructure are analyzed: Fibonacci laminated composite, wavy multilayered and bimetallic composite with thickness variation. The Fibonacci structure is analyzed as a quasi-periodic structure and the effective coefficients are obtained and compared with experimental and theoretical approaches. The effective coefficients for wavy laminated composite are calculated and different behavior of the interface (soft/hard) is considered. In order to validate the model a bimetallic composite with thickness variation is dealt and the nonzero effective coefficients are determinate using AHM and FEM. The described methodology is used to determinate the effective properties of the aorta and this artery is studied as a laminated shell composite. The effective coefficients for healthy

Table $7 \widehat{h}^{131}, \widehat{h}^{223}$ and second $\widehat{h}^{11}, \widehat{h}^{33}$ order homogenized coefficients for different values of $r$.

\begin{tabular}{lllll}
\hline$r$ & $\hat{h}^{131}$ & $\widehat{h}^{223}$ & $\widehat{h}^{11}$ & $\widehat{h}^{33}$ \\
\hline 1.02 & 1.249 & 68.412 & -4.899 & 71.670 \\
1.06 & 1.202 & 70.970 & -4.536 & 67.067 \\
1.1 & 1.158 & 73.533 & -4.212 & 62.288 \\
1.14 & 1.118 & 76.100 & -3.922 & 57.332 \\
1.18 & 1.080 & 78.670 & -3.661 & 52.199 \\
1.22 & 1.044 & 81.243 & -3.424 & 46.889 \\
\hline
\end{tabular}

Table 6

Comparison between AHM and FEM of the effective coefficients of the aorta for three and four layers.

\begin{tabular}{|c|c|c|c|c|c|c|c|c|c|}
\hline Methods & $C_{e}^{11}$ & $C_{e}^{12}$ & $C_{e}^{13}$ & $C_{e}^{22}$ & $C_{e}^{23}$ & $C_{e}^{33}$ & $C_{e}^{44}$ & $C_{e}^{55}$ & $C_{e}^{66}$ \\
\hline \multicolumn{10}{|c|}{ Three layers } \\
\hline AHM & 55.275 & 65.418 & 64.537 & 86.125 & 80.739 & 84.035 & 1.647 & 1.270 & 1.775 \\
\hline FEM & 55.292 & 65.428 & 64.546 & 86.122 & 80.737 & 84.032 & 1.647 & 1.293 & 1.775 \\
\hline \multicolumn{10}{|c|}{ Four layers } \\
\hline AHM & 10.488 & 7.844 & 6.602 & 8.799 & 5.384 & 5.604 & 0.109 & 0.150 & 0.781 \\
\hline FEM & 10.487 & 7.843 & 6.601 & 8.795 & 5.383 & 5.602 & 0.109 & 0.150 & 0.780 \\
\hline
\end{tabular}


(three layers) and unhealthy (four layers) aorta are computed and both cases are validated with FEM.

\section{Acknowledgments}

The funding of CONACYT project number 129658 and Coordinación de la Investigación Científica COIC-STIA-306-15(71787179) de la UNAM is gratefully acknowledged. The authors gratefully acknowledge to the project SHICHAN, supported by FSP (Cooperation Scientifique Franco-Cubaine) PROJET N 29935XH and to the project Composite Materials from University of Havana. Author AC acknowledges the financial support received through CNPQ (PQ 392298/2012-6). The author RRR wants to thank the PROPPI and Department of International Relations - DRI - Universidade Federal Fluminense UFF, for supporting the development of this work during his stay in Computer Institute of Universidade Federal Fluminese (Project Edital PI-UFF 2014).Thanks to Departamento de Matemáticas y Mecánica, IIMAS-UNAM, for its support and Ramiro Chávez Tovar and Ana Pérez Arteaga for computational assistance.

\section{Appendix A. Nonzero coefficients for a laminated composite with orthogonal coordinates}

We consider a laminated composite, where the orthogonal coordinate system is used to describe the geometry. The function $\varrho: \mathbb{R}^{2} \rightarrow \mathbb{R}$ is a stratified function of two variables $\varrho \equiv \varrho\left(x_{1}, x_{2}\right)$. The effective elastic tensor presented in Ref. [13] is a particular case of a function $\varrho$ in a rectangular coordinate system.

Using Equation (27), the nonzero effective coefficients can be written and the homogenized structure has monoclinic behavior.

$$
\begin{aligned}
& C_{e}^{11}=\left\langle C^{11}+C^{11} \frac{\partial \varrho}{\partial x_{1}} \frac{\partial N_{(1) 1}^{1}}{\partial y}+C^{12} \frac{\partial \varrho}{\partial x_{2}} \frac{\partial N_{(1) 2}^{1}}{\partial y}\right\rangle, \\
& C_{e}^{12}=\left\langle C^{12}+C^{11} \frac{\partial \varrho}{\partial x_{1}} \frac{\partial N_{(1) 1}^{2}}{\partial y}+C^{12} \frac{\partial \varrho}{\partial x_{2}} \frac{\partial N_{(1) 2}^{2}}{\partial y}\right\rangle, \\
& C_{e}^{13}=\left\langle C^{13}+C^{11} \frac{\partial \varrho}{\partial x_{1}} \frac{\partial N_{(1) 1}^{3}}{\partial y}+C^{12} \frac{\partial \varrho}{\partial x_{2}} \frac{\partial N_{(1) 2}^{3}}{\partial y}\right\rangle,
\end{aligned}
$$

$C_{e}^{16}=\left\langle C^{11} \frac{\partial \varrho}{\partial x_{1}} \frac{\partial N_{(1) 1}^{6}}{\partial y}+C^{12} \frac{\partial \varrho}{\partial x_{2}} \frac{\partial N_{(1) 2}^{6}}{\partial y}\right\rangle$,

$C_{e}^{22}=\left\langle C^{22}+C^{12} \frac{\partial \varrho}{\partial x_{1}} \frac{\partial N_{(1) 1}^{2}}{\partial y}+C^{22} \frac{\partial \varrho}{\partial x_{2}} \frac{\partial N_{(1) 2}^{2}}{\partial y}\right\rangle$,

$C_{e}^{23}=\left\langle C^{23}+C^{21} \frac{\partial \varrho}{\partial x_{1}} \frac{\partial N_{(1) 1}^{3}}{\partial y}+C^{22} \frac{\partial \varrho}{\partial x_{2}} \frac{\partial N_{(1) 2}^{3}}{\partial y}\right\rangle$,

$C_{e}^{26}=\left\langle C^{21} \frac{\partial \varrho}{\partial x_{1}} \frac{\partial N_{(1) 1}^{6}}{\partial y}+C^{22} \frac{\partial \varrho}{\partial x_{2}} \frac{\partial N_{(1) 2}^{6}}{\partial y}\right\rangle$,

$C_{e}^{33}=\left\langle C^{33}+C^{31} \frac{\partial \varrho}{\partial x_{1}} \frac{\partial N_{(1) 1}^{3}}{\partial y}+C^{32} \frac{\partial \varrho}{\partial x_{2}} \frac{\partial N_{(1) 2}^{3}}{\partial y}\right\rangle$,
$C_{e}^{36}=\left\langle C^{13} \frac{\partial \varrho}{\partial x_{1}} \frac{\partial N_{(1) 1}^{6}}{\partial y}+C^{23} \frac{\partial \varrho}{\partial x_{2}} \frac{\partial N_{(1) 2}^{6}}{\partial y}\right\rangle$,

$C_{e}^{44}=\left\langle C^{44}+C^{44} \frac{\partial \varrho}{\partial x_{2}} \frac{\partial N_{(1) 3}^{4}}{\partial y}\right\rangle$,

$C_{e}^{45}=\left\langle C^{44} \frac{\partial \varrho}{\partial x_{2}} \frac{\partial N_{(1) 3}^{5}}{\partial y}\right\rangle$,

$C_{e}^{55}=\left\langle C^{55}+C^{55} \frac{\partial \varrho}{\partial x_{1}} \frac{\partial N_{(1) 3}^{5}}{\partial y}\right\rangle$

$C_{e}^{66}=\left\langle C^{66}+C^{66} \frac{\partial \varrho}{\partial x_{2}} \frac{\partial N_{(1) 1}^{6}}{\partial y}+C^{66} \frac{\partial \varrho}{\partial x_{1}} \frac{\partial N_{(1) 2}^{6}}{\partial y}\right\rangle$.

\section{References}

[1] Fessel G, Broughton J, Fellows N, Durodola J, Hutchinson A. Evaluation of different lap-shear joint geometries for automotive applications. Int J Adhes Adhes 2007:27:574-83.

[2] Chen Y, Chiparus O, Sun L, Negulescu I, Parikh D, Clamari T. Natural fibers for automotive nonwoven composites. J Ind Text 2005;35:47-62.

[3] Xiong J, Ghosh R, Ma L, Vaziri A, Wang Y, Wu L. Sandwich-walled cylindrical shells with lightweight metallic lattice truss cores and carbon fiber-reinforced composite face sheets. Compos Part A 2014;56:226-38.

[4] Scarponi C. Hemp fiber composites for the design of a Naca cowling for ultralight aviation. Compos Part B 2015;81:53-63.

[5] Zhou Y, Lu Z, Yang Z. Progressive damage analysis and strength prediction of 2d plain weave composites. Compos Part B 2013;47:220-9.

[6] Gager J, Pettermann H. Numerical homogenization of textile composites based on shell element discretization. Compos Sci Technol 2012;72:806-12.

[7] Gager J, Pettermann H. Fem modeling of multilayered textile composites based on shell elements. Compos Part B 2015;77:46-51.

[8] Sofiyev A. Large-amplitude vibration of non-homogeneous orthotropic composite truncated conical shell. Compos Part B 2014;61:365-74.

[9] Sofiyev A. Buckling of heterogeneous orthotropic composite conical shells under external pressures within the shear deformation theory. Compos Part B 2016;84:175-87.

[10] Pobedrya B. Mechanics of composite materials. Moscow, Russia: Moscow State University Press; 1984.

[11] Bakhvalov N, Panasenko G. Homogenisation: averaging processes in periodic media, mathematics and its application (Soviet Series). 1989.

[12] Saha G, Kalamkarov AL, Georgiades AV. Asymptotic homogenization modeling and analysis of the effective properties of smart composite reinforced and sandwich shells. Int J Mech Sci 2007;49:138-50.

[13] Tsalis D, Chatzigeorgiou G, Charalambakis N. Homogenization of structures with generalized periodicity. Compos Part B 2012;43:2495-512.

[14] Murat F, Tartar L. H-convergence in topics in the mathematical modelling of composite materials. Progress in nonlinear differential equations and their applications, vol. 31; 1997. p. 21-43.

[15] Lutoborski A. Homogenization of linear elastic shells. J Elast 1985;15:69-87.

[16] Ciarlet PG. Mathematical elasticity: theory of shells, vol. III. Amsterdam, The Netherlands: Elsevier Science B. V.; 2000.

[17] Mohammad SQ, Ebrahim A, Wnchao W. Review of recent literature on static analyses of composite shells: 2000-2010. Open J Compos Mater 2012;2: 61-86.

[18] Challagulla K, Georgiades A, Saha G, Kalamkarov A. Micromechanical analysis of grid-reinforced thin composite generally orthotropic shells. Compos Part B 2008;39:627-44.

[19] Saha G, Kalamkarov AL, Georgiades AV. Effective elastic characteristics of honeycomb sandwich composite shells made of generally orthotropic materials. Compos Part A 2007;38:1533-46.

[20] Ossadzow-David C, Touratier M. A multilayered piezoelectric shell theory. Compos Sci Technol 2004;64:2121-37.

[21] Peng X, Cao J. A dual homogenization and finite element approach for material characterization of textile composites. Compos Part B Eng 2002:33:45-56.

[22] Liu M, Yu J. Finite element modeling of delamination by layerwise shell element allowing for interlaminar displacements. Compos Sci Technol 2003;63:517-29. 
[23] Li B, Li Y, Su J. A combined interface element to simulate interfacial fracture of laminated shell structures. Compos Part B 2014:58:217-27.

[24] Tsalis D, Baxevanis T, Chatzigeorgiou G, Charalambakis N. Homogenization of elastoplastic composites with generalized periodicity in the microstructure. Int J Plasticity 2013;51:161-87.

[25] Cabreras-Fernández D, Niazy AM, Kurtz RM, Djotiyan GP, Juhasz T. Finite element analysis applied to cornea reshaping. J Biomed Opt 2005;10(6): 064018.

[26] Pandolfi A, Holzapfel GA. Three-dimensional modeling and computational analysis of the human cornea considering distributed collagen fibril orientations. J Biomech Eng 2008;130:061006.

[27] Mahanth-Kasavajhala AR. Fracture analyses of aging aircraft structures and human aorta [Ph.D. thesis]. University of Nebraska-Lincoln; 2011.

[28] Ramirez M, Nava-Gomez G, Sabina F, Camacho-Monte H, Guinovart-Diaz R, Rodriguez-Ramos R, et al. Enhancement of young's moduli and auxetic windows in laminates with isotropic constituents. Int J Eng Sci 2012;58: 95-114.
[29] Bravo-Castillero J, Rodriguez-Ramos R, Mechkour M, Otero J, Sabina F. Homogenization of magneto-electro-elastic multilaminated materials. Q J Mech Appl Math 2008;61:311-32.

[30] Bravo-Castillero J, Otero J, Rodriguez-Ramos R, Bourgeat A. Asymptotic homogenization of laminated piezocomposite materials. Int J Solids Struct $1998 ; 35: 527-41$

[31] Xia H, Zhang XK, Hu A, Jiang SS, Peng RW, Zhang W, et al. Effective elastic constants and phonon spectrum in metallic ta/al quasiperiodic superlattices. Phys Rev B 1993:47(7):3890-5.

[32] Dumont S, Lebon F, Ould-Khaoua A. A numerical tool for periodic heterogeous media, application to interface in al/sic composite. J Appl Mech 2000;67(1): 214-7.

[33] Fish J, Belytschko T. A first course in finite elements. J. Wiley \& Sons; 2007.

[34] Dumont S, Lebon F, Rizzoni R. An asymptotic approach to the adhesion of thin stiff films. Mech Res Commun 2014;58:24-35.

[35] Gao F, Wantenabe M, Matsusawa T. Stress analysis in a layered aortic arch model under pulsatile blood flow. Biomed Eng Online 2006;5. 